\title{
Review Article \\ Correlates of Protection for M Protein-Based Vaccines against Group A Streptococcus
}

\author{
Shu Ki Tsoi, ${ }^{1}$ Pierre R. Smeesters, ${ }^{1,2}$ Hannah R. C. Frost, ${ }^{1}$ \\ Paul Licciardi, ${ }^{3,4}$ and Andrew C. Steer ${ }^{1,2,4}$ \\ ${ }^{1}$ Group A Streptococcus Research Group, Murdoch Childrens Research Institute, Melbourne, VIC 3052, Australia \\ ${ }^{2}$ Centre for International Child Health, University of Melbourne, Melbourne, VIC 3052, Australia \\ ${ }^{3}$ Pneumococcal Research Group, Murdoch Childrens Research Institute, Melbourne, VIC 3052, Australia \\ ${ }^{4}$ Department of Paediatrics, Royal Children's Hospital, Melbourne, VIC 3052, Australia
}

Correspondence should be addressed to Andrew C. Steer; andrew.steer@rch.org.au

Received 9 February 2015; Revised 28 April 2015; Accepted 3 May 2015

Academic Editor: Peirong Jiao

Copyright (C) 2015 Shu Ki Tsoi et al. This is an open access article distributed under the Creative Commons Attribution License, which permits unrestricted use, distribution, and reproduction in any medium, provided the original work is properly cited.

\begin{abstract}
Group A streptococcus (GAS) is known to cause a broad spectrum of illness, from pharyngitis and impetigo, to autoimmune sequelae such as rheumatic heart disease, and invasive diseases. It is a significant cause of infectious disease morbidity and mortality worldwide, but no efficacious vaccine is currently available. Progress in GAS vaccine development has been hindered by a number of obstacles, including a lack of standardization in immunoassays and the need to define human correlates of protection. In this review, we have examined the current immunoassays used in both GAS and other organisms, and explored the various challenges in their implementation in order to propose potential future directions to identify a correlate of protection and facilitate the development of $M$ protein-based vaccines, which are currently the main GAS vaccine candidates.
\end{abstract}

\section{Introduction}

Group A streptococcus (GAS, otherwise known as Streptococcus pyogenes) is one of the most important causes of infectious disease morbidity and mortality, with an estimated prevalence of severe disease of at least 18.1 million cases leading to more than 500,000 deaths annually [1]. Much of the burden exists in less developed countries where control strategies are difficult to implement and ensure efficacy. A vaccine is therefore widely acknowledged as an important strategy to reduce the burden of GAS disease globally. Several vaccine candidates are presently in various stages of preclinical development [2], and a limited number have also reached early phase clinical trials $[3,4]$. A key element in vaccine development is the availability of a validated and standardized immunoassay that correlates with immune protection. However, despite decades of research, there is no single standardized immunoassay and certainly no human correlate of protection (CoP) established for GAS [5]. In the case of other bacterial pathogens, such as Streptococcus pneumoniae and group B streptococcus (GBS), for which vaccines are widely available or development is underway, CoPs provide a means of assessing the true efficacy and immunogenicity of potential vaccines $[6,7]$.

\section{Immune Response to GAS Infection}

A solid understanding of bacterial pathogenesis and host immune response lays the foundations for robust CoPs. It is widely agreed that the $\mathrm{M}$ protein is an important virulence factor of GAS, conferring both adhesion and antiphagocytic properties through binding of various host proteins and interacting with the complement pathway $[8,9]$. The $M$ protein is an alpha-helical coiled-coil dimer extending from the surface of the bacteria as a fibril [10]. Its structure is divided into conserved, central variable, and N-terminal hypervariable regions [11]. Some $M$ proteins may have a nonhelical portion at the distal end of the $\mathrm{N}$-terminal region, 
but the significance of this is unknown [9]. There are a number of $A, B, C$, and $D$ repeats that vary between the different $M$ proteins, with increasing sequence conservation downstream of the hypervariable region.

The current GAS classification system, known as emmtyping, is based on sequencing of $10-15 \%$ of the emm gene (which encodes the M protein) with $223 \mathrm{emm}$-types identified worldwide $[12,13]$. Recently, a new adjunct method of classification, known as the "emm-cluster system" has been developed, which organizes the $223 \mathrm{emm}$-types into 48 distinct emm-clusters based on the phylogenetic analysis of the whole $\mathrm{M}$ protein and its structural and binding properties [14-16].

The main immune mechanisms against GAS are thought to be antibody-mediated. Studies in mice have demonstrated that mucosal IgA antibodies against the M protein prevent colonization and adherence of GAS $[17,18]$. Binding of typespecific IgG antibodies to the $\mathrm{N}$-terminus of the $\mathrm{M}$ protein in the host activates the complement pathway, leading to deposition of C3b and facilitating phagocytosis and killing [18]. Limited evidence suggests that these type-specific antibodies provide long-lasting protection against homologous strains [19]. Whilst the conserved region of the M protein has not been as extensively studied as the N-terminus, it has also been shown to elicit osonophagocytic antibodies [2023]. Antibodies produced against the $B$ repeat region of the $\mathrm{M}$ protein are not thought to be opsonic and thus unlikely to be protective [24].

Memory B cells and T cells also appear to play a role in propagating immunity [21], and several immunodominant $\mathrm{T}$ cell epitopes have been mapped [18], although the exact mechanisms of cell-mediated immunity in GAS are still unclear [21, 23]. Non-M protein virulence factors such as C5a peptidase, lipoteichoic acid, protein F, and Streptococcal fibronectin-binding protein have been shown in animal studies to raise protective antibodies that contribute to host immunity, particularly in preventing colonization [18, 25]. Antibodies to streptococcal toxins such as streptococcal pyrogenic exotoxins $\mathrm{A}, \mathrm{B}$, and $\mathrm{C}$ and streptococcal erythrogenic exotoxin $\mathrm{B}$ have also been proposed to play a role in in vivo immunity [18]. Other potentially immunogenic antigens that have been investigated as vaccine candidates include streptococcal protective antigen, serum opacity factor, streptococcal pili, S. pyogenes cell envelope protease, and GAS carbohydrate [25]. Whilst all of the above may play a role in GAS immunity, it is believed that opsonic type-specific antibodies remain responsible for clearing infection [18].

The exact natural history of immunity against GAS infections also remains unclear, although there are distinct peaks of increased incidence at different age groups for each GAS disease (Figure 1). For instance, the incidence of GAS superficial infections is high in children but decreases in adulthood [20]. Anti-GAS antibody levels in adults are much higher compared with children, suggesting that this natural acquisition of antibodies due to exposure over time provides protective immunity against GAS diseases later in life [20, 26]. Similarly for severe and invasive GAS diseases, there is decreased incidence in adulthood; the significant peak

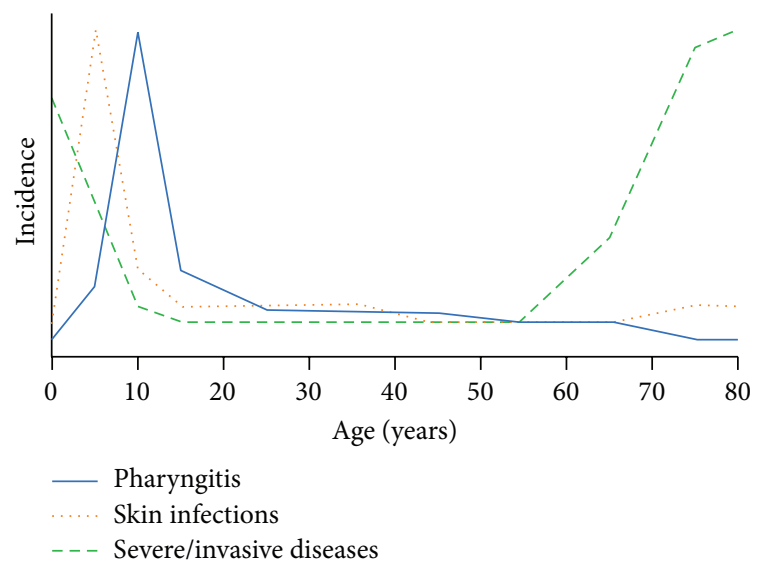

FIGURE 1: Schematic representation of incidence of group A streptococcal diseases by age using data from epidemiological reports [27, 30-33].

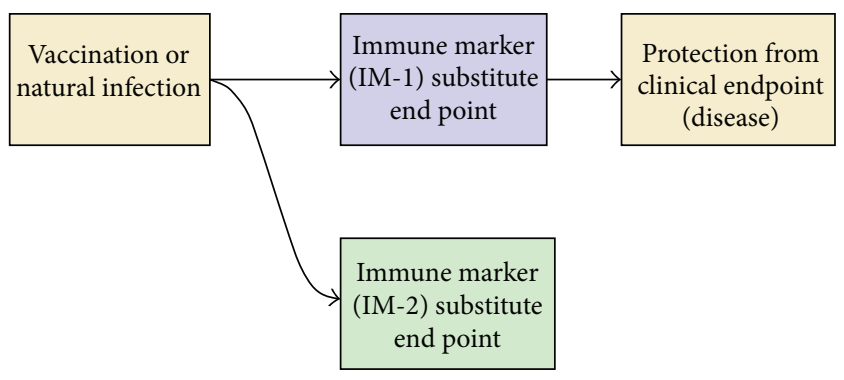

FigURE 2: Process of immunity and correlates of protection. Immune markers 1 and 2 (IM-1, IM-2) are correlates of protection, but only IM-1 is a surrogate. Arrows imply direct causal relationships. Figure adapted from WHO [34].

in the elderly is likely to be due to comorbid illness and immunosenescence [27-29].

\section{Immune Correlates of Protection}

3.1. Definitions. A "correlate" is "an attribute that is statistically associated with an endpoint (without the association necessarily being causal)," whilst a "surrogate" or "mechanistic CoP" (mCoP) has the added criterion of being part of the causal pathway and the mechanism by which a vaccine induces protection (Figure 2) [34].

3.2. Rationale for Establishing Correlates of Protection in GAS. In 2011, it was agreed that a roadmap for GAS vaccine development was needed to harness the efforts of the international community and two key components were identified as (1) definition of human CoPs for GAS and (2) development of high throughput standardized assays that accurately represent in vivo immunity $[5,25,35]$.

A key long-term advantage of CoPs is in enabling vaccine licensure by obviating the need for demonstrating field efficacy in the scenario where efficacy trials are logistically or ethically challenging [36]. Establishing the efficacy and 
TABLE 1: Advantages and disadvantages of different immunoassays currently utilized in GAS research.

\begin{tabular}{lll}
\hline Immunoassay & Advantages & Disadvantages \\
\hline ELISA & $\begin{array}{l}\text { High-throughput } \\
\text { Easily standardized } \\
\text { Reproducible }\end{array}$ & May measure non-functional antibodies \\
\hline Bactericidal assays & Measures functional antibodies & $\begin{array}{l}\text { Labour-intensive } \\
\text { Inter-assay variability } \\
\text { Use of whole human blood imposes two hour time restriction } \\
\end{array}$ \\
\hline
\end{tabular}

safety of vaccine candidates can be a lengthy and expensive endeavour involving large-scale phase III trials. Whilst it is likely that the first GAS vaccine candidate to be licensed will be approved based on phase III trials using pharyngitis as an end-point, CoPs may provide an alternative for second generation vaccines. If an immunological marker is established as a $\mathrm{CoP}$ and it is demonstrated that a vaccine candidate meets or exceeds an immune threshold, then it may be approved based on serological data alone [34]. Successful precedents include vaccines for meningococcal serogroups B [37] and C [38-40], influenza [41], S. pneumonia [42], and Haemophilus influenzae type b (Hib) [43]. Not only can CoPs fast-track the licensure process and potentially reduce costs, but they can also be useful when placebo randomized controlled trials (RCT) are no longer possible or ethical (as in the case of Hib) [34]. This may be the case if a successful GAS vaccine is developed; it may be unethical to allow cases of RHD and potentially fatal invasive diseases to occur in subsequent vaccine development trials when a vaccine would prevent them.

Correlates of protection may also be used to inform seroepidemiology studies and surveillance of populations postvaccination. This could be relevant to GAS because of high global diversity of strains that may evoke different patterns of immune responses and to evaluate whether a vaccine will introduce pressure that leads to the emergence of new strains. Further applications that could be explored include passive protection, where the $\mathrm{CoP}$ is used to determine the amount of antibody to administer in passive immunizations and as screening or diagnostic tools, for instance, in testing antibody levels against rubella for pregnant women [34].

Thus, establishing a CoP would provide an adjunct tool of great benefit in GAS vaccine development [6]. However, numerous challenges have hindered CoP development for GAS. Firstly, there are technical difficulties in developing a high quality, standardized immunoassay for GAS. Further, the utilization of the assay to determine a CoP for GAS raises its own set of challenges in terms of study design and use of animal or human models.

\section{Development of a Standardized Immunoassay for GAS}

The optimal immunoassay used to define CoPs should ideally meet several criteria. It should be reproducible across different laboratories with a common standard operating protocol and equivalent data sets. For GAS, one of the key points will be ensuring that antigens and GAS strains used by different laboratories are comparable. The methodology needs to be based on a thorough understanding of GAS pathogenesis, host interactions, and immune response in order to be clinically relevant [36]. A practical assay should have a high throughput.

It is worth noting that these criteria for the establishment of a robust standardized immunoassay in GAS are important not only in terms of defining a CoP, but also in securing reliable immunological data for the conventional aspects of vaccine development to enable proof-of-concept studies, successful progression through trial phases, and ensuring ongoing funding from the relevant sources. A robust immunoassay will also be particularly critical in helping to overcome the specific challenges in GAS vaccine development such as ensuring coverage across the many different strains and variation in geographical distribution.

There are currently two main types of immunoassays utilized in GAS research: assays that give a purely quantitative measurement of antibody such as the enzyme-linked immunosorbent assay (ELISA) and functional bactericidal assays that demonstrate immunity through evidence of phagocytic killing of GAS (Table 1). Assays that have been established for other pathogens that have potential application to GAS include those for $S$. pneumoniae and GBS [6]. The opsonophagocytic assays proposed as CoPs may not necessarily apply to all GAS protective antigens.

4.1. Enzyme-Linked Immunosorbent Assay. The ELISA has been used for quantification of antibodies in GAS in the development of all the major GAS vaccine candidates in testing of both rabbit and mice antisera, as well as human antisera in two phase I trials $[4,17,21,23,44-49]$. The ELISA has been used successfully in $S$. pneumoniae research to establish CoPs [6] and in GBS to propose potential CoPs values [7], suggesting that the ELISA may be a good candidate for determining GAS CoPs.

One issue is the choice of antigen to use, such as recombinant $\mathrm{M}$ protein [17], or synthetic peptides [4, 44, 47]. The peptide method is generally favoured in preclinical studies as it allows for the testing of antibodies specific to the vaccine epitope only and minimizes the amount of nonspecific antibodies detected [10, 44, 47]. However, there is a theoretical concern that the folding structure of peptides may be altered from the normal structure when within 
TABLE 2: Variations across methods used in performing the indirect bactericidal test.

\begin{tabular}{llr}
\hline Assay component & Variations & Reference(s) \\
\hline Bacteria growth phase & Stationary & {$[56]$} \\
& $($ Mid)logarithmic & {$[44-47]$} \\
\hline \multirow{3}{*}{ Bacterial dilution } & $10^{-4}$ with $1: 4$ serial dilution & {$[56]$} \\
& $10^{-4}$ with $1: 7$ serial dilution & {$[46,47,57]$} \\
\hline & $10^{-5}$ with $1: 4$ serial dilution & {$[44-47]$} \\
Growth media & Todd-Hewitt broth only & {$[56]$} \\
& Todd-Hewitt broth + 20\% normal calf/horse serum & {$[57]$} \\
\hline Serum & Serum broth & {$[56]$} \\
\hline
\end{tabular}

a whole $\mathrm{M}$ protein and consequently give results that may not be clinically relevant [50].

There are potential methods to increase specificity for ELISAs using the full M protein. For example, in S. pneumoniae research, initial ELISAs were found to correlate poorly with in vivo reactions due to measurement of nonfunctional antibodies to pneumococcal cell wall polysaccharide (C-PS) in addition to anti-capsular antibodies [51]. This problem was overcome by preabsorption of serum with highly purified C-PS before use in the ELISA, to remove non-functional antibodies [51]. Similarly, preabsorption with pneumococcal type $22 \mathrm{~F}$ capsular polysaccharide neutralized antibodies cross-reactive to common epitopes, leaving only serotypespecific antibodies to be measured in the ELISA [52]. For GAS, the addition of a preabsorption step with an unrelated $M$ peptide or full $M$ protein could increase the specificity and detection of functional antibodies. A slightly different approach was taken in GBS, where alterations to the antigen were made $[53,54]$. The GBS III polysaccharide was mixed with methylated human serum albumin (HSA) to increase adherence of the antigen to the microtitre plates and coupled with biotin or nonmethylated HAS [53], and a subsequent study found using nonmethylated HSA produced the highest specificity and sensitivity [55]. In GAS, mixing the full $\mathrm{M}$ protein with fibrinogen or HSA to bind the $\mathrm{B}$ repeat and $\mathrm{C}$ repeat portions of the $M$ protein, respectively, could reduce detection of non-functional antibodies.

A high quality ELISA should also be reproducible and standardized across laboratories. There is currently no standardized protocol between GAS laboratories, making it difficult to confidently compare results between studies. Two approaches could potentially be used to ameliorate this issue: (1) attempting to regulate all steps and reagents used or (2) providing a standard serum for laboratories to use as a reference. The latter is utilized for S. pneumoniae, where ELISAs are standardized against the reference serum, $007 \mathrm{sp}$, created from volunteers immunized with the 23valent vaccine [58]. This allows for the continued comparison of assay results in vaccine evaluations.

4.2. Multiplexed Flow Cytometry Assays. Martins et al. took a novel approach by developing a multiplexed fluorescent immunoassay using Luminex Multi-Analyte Profiling technology (Luminex Corp., Austin, TX) [59]. IgG antibody responses against nine common GAS antigens (including the $M$ protein) conjugated to fluorescent microspheres were simultaneously measured using either human sera or animal antibodies. Whilst the assay quantified antibody responses to antigens in a high throughput fashion, a high degree of nonspecific binding of IgG to microspheres was reported and no comparison with ELISA or bactericidal assays was performed.

4.3. Bactericidal Assays. The principle of GAS bactericidal assays is to incubate whole blood with bacterial inoculum and then determine bactericidal activity by measuring colonyforming units (CFU). There are two variations, the direct bactericidal test (DBT) and the indirect bactericidal test (IBT). The DBT utilizes the test subject's whole blood as a source for serum and phagocytes. The IBT only uses serum from the test subject and nonimmune human blood from a separate donor as the source of phagocytes, with the aim of reducing variability as donors are screened to ensure that there are no type-specific or cross-reactive antibodies present [56]. This also allows for testing of animal sera [56]. The IBT has lower sensitivity with low antibody titres, leading to the development of an optional preopsonization step where antisera is first incubated with GAS to increase antibody binding before adding the source of phagocytes [56]. Currently, the IBT is the most commonly used method to assess functional immunity to GAS $[4,44-48]$. Despite this, there are still many variations in methodology between laboratories (Table 2), and even in the World Health Organization established protocols, two main methods of performing the IBT are described [56].

Inter-assay variability is high because of the different methodologies and because of the unstandardized element of donor blood. The need for fresh human blood is problematic as it means that assays need to be performed within two hours of venepuncture. Both the donor and test subject must have the same blood group, or only group O blood can be used, to prevent an ABO incompatibility reaction [56]. The IBT is labour intensive and low throughput, making it a less suitable choice for determining a CoP for GAS.

A variation of the IBT using light microscopy of Wright smear stains to define opsonization rather than counting CFU 
has also been used to test GAS vaccine candidates $[46,60]$. However, in the phase I trial of the hexavalent GAS vaccine, less of a response was seen in the IBT when compared to this version of the opsonophagocytic assay [46], suggesting that in some cases association of bacteria with the cell wall of leukocytes may not equate to killing.

4.4. HL-60 Opsonophagocytic Assay. The HL-60 opsonophagocytic assay (OPA) is an alternative functional opsonic assay that could potentially be utilized in GAS, given its prior success with S. pneumonia [61] and GBS [62]. The HL-60 cells used as the source of phagocytes are human promyelocytic leukaemia cells capable of being cultured and differentiated, thus eliminating the need for human donors and potentially decreasing inter-assay variability. The assay tests the bactericidal activity and ability of sera to opsonize viable bacteria in the presence of differentiated HL60 neutrophils and complement.

4.5. Streptococcus pneumoniae HL-60 OPA. Romero-Steiner et al. first developed the HL-60 OPA [61] to measure functional antibodies against $S$. pneumoniae. They demonstrated for seven serotypes that the HL-60 OPA correlated highly with OPAs using peripheral blood leukocytes (PBL), the previous standard for effector cells [61]. Over time, there have been a number of variations trialled and modifications made to the assay (Table 3 ).

The HL-60 OPA was tested in various multilaboratory trials using multiplexing and automated colony counting, showing robust inter-assay and interlaboratory reproducibility and validation $[63,64]$, and a standardized protocol is now available online [65]. However, it was found that the execution of the OPA is not always tightly regulated across all laboratories, and whilst overall agreement is sufficient, there remains higher variability compared to ELISA [66]. Further, the correlation between ELISA, HL-60, and PBL OPAs is poor for some specific pneumococcal serotypes. For example, five serum samples tested for serotype $19 \mathrm{~F}$ immunity had elevated ELISA IgG titres but no opsonophagocytic activity [61]. This may in fact be a problem with the ELISA; a study comparing ELISA and OPA in children with human immunodeficiency virus (HIV) in South Africa found raised ELISA antibody titres in both infected and noninfected groups, but lower OPA titres in HIV-infected individuals, suggesting that the ELISA could be detecting non-protective antibodies [67].

Nevertheless, evidence of bactericidal activity of functional antibodies is desired when considering licensure of a new vaccine candidate, and thus the WHO has determined for S. pneumoniae conjugate vaccines that an OPA titre of $1: 8$ in conjunction with an ELISA antibody concentration of at least $0.35 \mu \mathrm{g} / \mathrm{mL}$ demonstrates that a candidate provides sufficient protection to be considered for licensure [66].

It has been proposed that the use of the 007sp reference serum could also increase standardization [66]. The development of a reference serum for GAS would create a degree of uniformity across laboratories and help facilitate immunoassay and vaccine development. Furthermore, the establishment of a standard collection of GAS isolates for laboratories to reference would increase standardization in the performance of immunoassays used in vaccine studies.

4.6. Group B Streptococcus HL-60 OPA. The HL-60 OPA was adapted for use in GBS by validating it against the previous "gold standard" OPA that uses human PBLs [62]. Attempts to increase throughput of the assay have focussed on developing a fluorescent OPA (flOPA) using fluorescence activated cell sorting (FACS) to measure the uptake of fluorescently labelled, whole killed GBS cells, or antigencoated fluorescent microspheres [68]. The flOPA has multiple advantages including the ability to be multiplexed, the ability to standardize complement and effector cells, and a faster running time [69]. A criticism of previous flOPAs has been the inability to distinguish between adhered and internalized bacteria [68]. Fabbrini et al. have overcome this for GBS with pHrodo, a $\mathrm{pH}$-sensitive fluorogenic dye that only emits a bright red signal in an acidic environment [68]. This $\mathrm{pH}-$ dependent reaction means that pHrodo-labelled bacteria are only detected when they are in phagolysosomes. Bacteria that are bound to the HL-60 cell wall show only a very low signal on FACS, and thus the actual bactericidal antibodies can be measured rather than just measuring adhered antibodies. This method was shown to be reproducible and as sensitive and specific as a traditional OPA [68]. The potential applications of this technique are promising; however no studies using human sera have been carried out to date, and FACS remains an expensive method requiring skilled technicians.

\section{Implementing Immunoassays to Determine a Correlate of Protection}

Once a standardized immunoassay is established, studies can be carefully designed to determine what levels of immune markers correlate with protection.

5.1. Geographic Variation. A key challenge in establishing a $\mathrm{CoP}$ is the inherent heterogeneity present within populations and environments. There is a distinct variance in distribution of strains in developed countries when compared with resource poor regions such as Africa and the Pacific $[70,71]$. The highest rates of GAS diseases are in these low socioeconomic areas $[1,72]$, where crowding, poor hygiene, and tropical climate are likely to result in greater exposure and infection pressures. These differences may cause varying protective antibody levels for each population. Early studies suggested that different GAS strains require different levels of antibodies to be considered immune [19]. If that is the case, it may be difficult to accurately determine specific CoPs due to the challenges of obtaining comprehensive data from developing regions and the apparently constantly evolving epidemiological landscape $[1,70]$.

5.2. Host Factor Variation. Individual host factors can also alter CoPs, as a person's immunity changes with age and between individuals. The level of antibody that is protective in a young healthy adult may not protect against infection in an elderly individual [34]. GAS bactericidal antibodies have 


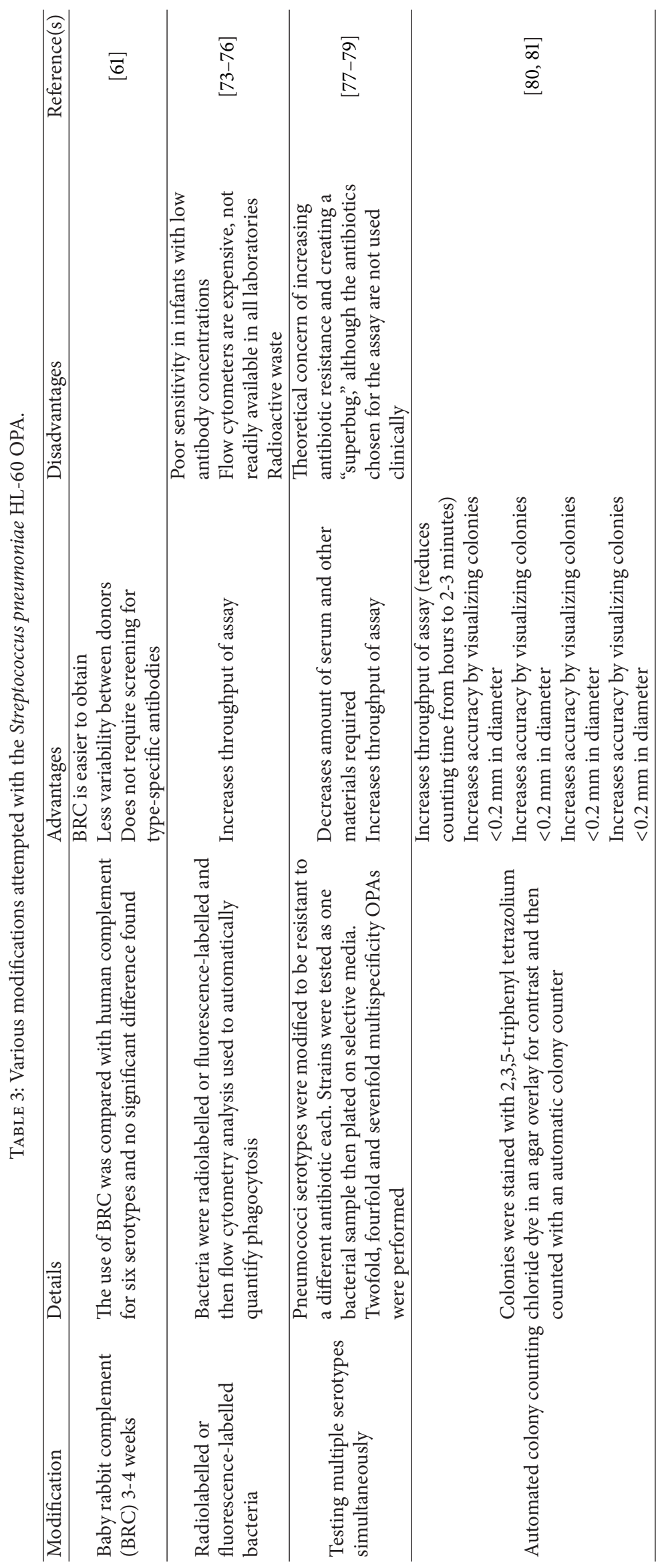


been shown to persist for up to 30 years but to varying degrees between persons [19], making it potentially difficult to predict CoPs in the long-term. Cell-mediated immunity involving $\mathrm{B}$ and $\mathrm{T}$ cells has also been shown to play a role in longterm memory. In particular, memory B cells are capable of mounting a rapid response to GAS infection in animal studies [21], potentially meaning that long-term immunity may be more dependent on an individual's cellular response and that low circulating antibody titres do not necessarily correlate with a lack of protection. For instance, the Hib conjugate vaccine was licensed based on a $\mathrm{CoP}$ of serum antibody concentration of $0.04-1.0 \mu \mathrm{g} / \mathrm{mL}$ but variations from this range have been shown in different studies to be protective, and it is thought that immunologic memory and population differences have not been accounted for [43].

5.3. Vaccine Composition and Mode of Delivery. The choice of antigen(s) for a vaccine candidate, route of administration, and immune marker targeted can also result in different CoPs. For instance, the protective titre required for typespecific antibodies raised by the 30 -valent vaccine [44] may be different from that raised by the J8-DT vaccine against the conserved region of the M protein [22]. Secretory IgA antibodies are part of mucosal protection and inhibit GAS colonization but may not always necessarily correlate with serum IgG levels or protection against invasive disease [17]. There is some evidence that mucosal IgA antibodies may be able to contribute to immunity in certain cases, with intranasal immunisation of mice shown to induce salivary antibodies and protect against subsequent intranasal challenge $[82,83]$. These studies raise the possibility that mucosally delivered vaccines (e.g., intranasal) would require separate development of mucosal assays and CoPs [36]. It is also unclear whether serum opsonophagocytic IgG antibodies involved in clearing infection are protective against recurrent skin infections, as most studies of immune protection against GAS have traditionally focused on pharyngitis.

5.4. Animal Models. Animal models provide a way of studying in vivo immunity and pathogenesis, and pathogens that have used animal models to determine CoPs include Clostridium tetani and Clostridium botulinum [34]. However, there have been difficulties in developing accurate disease models and bridging the gap from animal to human immune response because GAS is a human-only pathogen [84]. There have been numerous animal models of GAS infections developed, spanning from murine models for virulence and vaccine studies to RHD in rats; invasive disease in rabbits or pigs; and pharyngitis in primates [85]. Murine models have been used in studies of several vaccine candidates [47, 86]. However, there are several limitations to these models: only a select number of GAS strains are virulent in mice; repeated passage of strains may be needed but result in mutations altering virulence; colonization is often difficult to achieve; and true pharyngitis does not occur [85]. As such, murine models are unlikely to give an accurate indication of human CoPs for GAS. Nonhuman primate models have been established in an attempt to provide a more clinically relevant model of GAS pharyngitis [87]. Although there is variable reproducibility of the severity of clinical disease seen in monkeys, they have been shown to produce type-specific opsonic antibodies (measured by bactericidal assays) [87] and could be utilized in challenge or vaccine studies to evaluate CoPs.

5.5. Study Design. Traditionally, CoPs have been established through a RCT phase III vaccine trial as has been the case for Hib, tuberculosis, and S. pneumoniae vaccine candidates [34, 88]. Other studies nested within RCTs may also be used to inform CoPs, as demonstrated by a case-control study of the acellular pertussis vaccine [89]. The lack of phase III trials has hindered the establishment of CoPs for GAS as RCTs ideally allow for an understanding of the relationships between the vaccine, immune markers, and clinical endpoint (Figure 2) for CoPs to be determined with appropriate statistical power.

Similar challenges face the GBS vaccine field where there is no vaccine beyond phase II trials currently available. Lin et al. used a case-control study to estimate a $\mathrm{CoP}$ in order to facilitate vaccine licensure [7]. Maternal and cord serum samples from infants across 14 hospitals were compared by ELISA to establish a relationship between IgG levels against GBS and early-onset disease in neonates. In this study, an antibody titre $\geq 5 \mu \mathrm{g} / \mathrm{mL}$ was protective against neonatal disease [7]. A case-control study conducted by Baker et al. also evaluated maternal antibody levels required to protect against GBS early-onset disease, finding that an overall titre $\geq 1 \mu \mathrm{g} / \mathrm{mL}$ conferred a $70 \%$ risk reduction [90]. More specifically, it was identified that different antibody levels were found to be protective for the different GBS types Ia, III, and V [90]. There are limited data from human natural infection studies for a number of GAS vaccine candidates. For example, a study of serum IgG antibodies against p145, the parent peptide of $J 8$, showed an age-related increase in antibody titres suggesting that these antibodies may correlate with protection over the life course (Figure 1) [20]. Similarly, anti-C5a peptidase titres were found to be in significantly higher concentrations in adults than in children in Minnesota [91]. Further, a study of children aged 5-14 years in Mexico observed higher titres of anti-GAS carbohydrate antibodies that correlated with reduced colonization of the pharynx suggesting that there may be a threshold protective antibody titre [92]. These studies suggest that defining a $\mathrm{CoP}$ without data from phase III clinical trials will be challenging, although an important consideration in the future remains. Additionally, whilst this evidence is insufficient to establish a CoP, it may help to inform clinical "proof-of-concept," that is, determination of whether an intervention is biologically active or inactive [93]. Proof-of-concept is an important bridging step in ensuring that government bodies, investors, and pharmaceutical companies continue to support the vaccine development process and allow for larger scale efficacy studies where a CoP may then be established.

Data from phase I and II clinical trials are also relevant to the current status of GAS vaccine research as has been the case for phase II trials of the botulinum $\mathrm{F}$ toxoid vaccine [97] and the Meningococcal $\mathrm{C}$ conjugate vaccine in 
TABLE 4: Previous GAS human challenge studies. CFU/mL: colony-forming units per millilitre.

\begin{tabular}{ccccccc}
\hline Year & Strain & Dose $(\mathrm{CFU} / \mathrm{mL})$ & Number of participants & Number of not vaccinated & Clinical illness in nonvaccine group & Reference \\
\hline 1971 & M1 & $4 \times 10^{6}$ & 50 & 25 & $52 \%$ & {$[9 \%$} \\
1973 & M1 & $4 \times 10^{6}$ & 44 & 23 & {$[94]$} \\
1975 & M3, M12 & $5 \times 10^{6}$ & 84 & 36 & $44 \%$ & {$[95]$} \\
\hline
\end{tabular}

the United Kingdom [40]. In the case of Meningococcal C, protective titres extrapolated from animal studies were used to inform human CoPs, and vaccine licensure was approved by comparing CoPs with the preexisting vaccine [34].

5.6. Human Challenge Model. Given the limited clinical applicability of animal models and the potential drawbacks of phase III clinical trials, human challenge models would provide a controlled method for testing of new vaccines. Many pathogens including, but not limited to S. pneumoniae [98], cholera [99], malaria [100], and dengue virus [101], utilize human challenge models in their fields. Three pharyngeal challenge studies of GAS were carried out in the 1970s with a total of 178 volunteers (Table 4) [94-96]. Importantly, the trials were safe, with penicillin administered within the therapeutic timeframe and patients closely monitored to prevent progression into invasive disease or autoimmune sequelae. In a potential GAS human challenge model, individuals would be infected with a carefully predetermined virulent strain of GAS known to cause pharyngitis and followed through to see if they develop clinical disease. By analyzing blood samples taken at different time points and comparing immune markers with clinical outcomes, one could then establish potential CoPs.

\section{Conflict of Interests}

The authors declare that there is no conflict of interests regarding the publication of this paper.

\section{References}

[1] J. R. Carapetis, A. C. Steer, E. K. Mulholland, and M. Weber, "The global burden of group A streptococcal diseases," The Lancet Infectious Diseases, vol. 5, no. 11, pp. 685-694, 2005.

[2] Y. Song, X. Zhang, C. Lu, F. Zhang, and H. Zhu, "Progress in development of group A streptococcus vaccines," Current Pharmaceutical Biotechnology, vol. 14, no. 11, pp. 947-950, 2013.

[3] RHDAustralia, High Hopes for J8-DT 2013, 2014, http://www .rhdaustralia.org.au/news-events/newsroom/high-hopes-j8-dt.

[4] S. A. McNeil, S. A. Halperin, J. M. Langley et al., "Safety and immunogenicity of 26-valent group A Streptococcus vaccine in healthy adult volunteers," Clinical Infectious Diseases, vol. 41, no. 8, pp. 1114-1122, 2005.

[5] J. B. Dale, V. A. Fischetti, J. R. Carapetis et al., "Group A streptococcal vaccines: paving a path for accelerated development," Vaccine, vol. 31, no. 2, pp. B216-B222, 2013.

[6] S. Plotkin, "Vaccines: correlates of vaccine-induced immunity," Clinical Infectious Diseases, vol. 47, no. 3, pp. 401-409, 2008.

[7] F. Y. C. Lin, J. B. Philips III, P. H. Azimi et al., "Level of maternal antibody required to protect neonates against early-onset disease caused by group B Streptococcus type Ia: a multicenter, seroepidemiology study," Journal of Infectious Diseases, vol. 184, no. 8, pp. 1022-1028, 2001.

[8] M. J. Walker, T. C. Barnett, J. D. McArthur, J. N. Cole, C. M. Gillen, and A. Henningham, "Disease manifestations and pathogenic mechanisms of group A streptococcus," Clinical Microbiology Reviews, vol. 27, pp. 264-301, 2014.

[9] P. R. Smeesters, D. J. McMillan, and K. S. Sriprakash, "The streptococcal M protein: a highly versatile molecule," Trends in Microbiology, vol. 18, no. 6, pp. 275-282, 2010.

[10] MW. Cunningham, "Pathogenesis of group A streptococcal infections and their sequelae," in Hot Topics in Infection and Immunity in Children IV, vol. 609 of Advances in Experimental Medicine and Biology, pp. 29-42, Springer, New York, NY, USA, 2008.

[11] L. A. Marraffini, A. C. Dedent, and O. Schneewind, "Sortases and the art of anchoring proteins to the envelopes of grampositive bacteria," Microbiology and Molecular Biology Reviews, vol. 70, no. 1, pp. 192-221, 2006.

[12] D. J. McMillan, P.-A. Drèze, T. Vu et al., "Updated model of group A Streptococcus M proteins based on a comprehensive worldwide study," Clinical Microbiology and Infection, vol. 19, no. 5, pp. E222-E229, 2013.

[13] Centers for Disease Control and Prevention Streptococcus Laboratory, "Streptococcus pyogenes Blast-emm sequence database," http://www2a.cdc.gov/ncidod/biotech/strepblast .asp.

[14] M. Sanderson-Smith, D. M. P. De Oliveira, J. Guglielmini et al., "A systematic and functional classification of streptococcus pyogenes that serves as a new tool for molecular typing and vaccine development," Journal of Infectious Diseases, vol. 210, pp. 1325-1338, 2014.

[15] N. Baroux, E. D'Ortenzio, N. Amedeo et al., "The emmcluster typing system for Group A Streptococcus identifies epidemiologic similarities across the pacific region," Clinical Infectious Diseases, vol. 59, no. 7, pp. e84-e92, 2014.

[16] S. T. Shulman, R. R. Tanz, J. B. Dale, A. C. Steer, and P. R. Smeesters, "Added value of the emm-cluster typing system to analyze group A Streptococcus epidemiology in high-income settings," Clinical Infectious Diseases, vol. 59, no. 11, pp. 16511652, 2014.

[17] M. A. Hall, S. D. Stroop, M. C. Hu et al., "Intranasal immunization with multivalent group A streptococcal vaccines protects mice against intranasal challenge infections," Infection and Immunity, vol. 72, no. 5, pp. 2507-2512, 2004.

[18] M. W. Cunningham, "Pathogenesis of group a streptococcal infections," Clinical Microbiology Reviews, vol. 13, no. 3, pp. 470511, 2000.

[19] R. C. Lancefield, "Persistence of type-specific antibodies in man following infection with group A streptococci," The Journal of Experimental Medicine, vol. 110, no. 2, pp. 271-292, 1959.

[20] E. R. Brandt, W. A. Hayman, B. Currie et al., "Opsonic human antibodies from an endemic population specific for a conserved 
epitope on the M protein of group A streptococci," Immunology, vol. 89, no. 3, pp. 331-337, 1996.

[21] M. Pandey, M. N. Wykes, J. Hartas, M. F. Good, and M. R. Batzloff, "Long-term antibody memory induced by synthetic peptide vaccination is protective against streptococcus pyogenes infection and is independent of memory T Cell help," Journal of Immunology, vol. 190, no. 6, pp. 2692-2701, 2013.

[22] M. R. Batzloff, W. A. Hayman, M. R. Davies et al., "Protection against group a streptococcus by immunization with J8diphtheria toxoid: Contribution of J8- and diphtheria toxoidspecific antibodies to protection," The Journal of Infectious Diseases, vol. 187, no. 10, pp. 1598-1608, 2003.

[23] M. Pandey, M. R. Batzloff, and M. F. Good, "Mechanism of protection induced by group A Streptococcus vaccine candidate J8-DT: contribution of B and T-cells towards protection," PLOS ONE, vol. 4, no. 4, Article ID e5147, 2009.

[24] C. Sandin, F. Carlsson, and G. Lindahl, "Binding of human plasma proteins to Streptococcus pyogenes M protein determines the location of opsonic and non-opsonic epitopes," Molecular Microbiology, vol. 59, no. 1, pp. 20-30, 2006.

[25] A. C. Steer, J. B. Dale, and J. R. Carapetis, "Progress toward a global group A streptococcal vaccine," The Pediatric Infectious Disease Journal, vol. 32, no. 2, pp. 180-182, 2013.

[26] V. A. Fischetti, D. E. Bessen, O. Schneewind, and D. E. Hruby, "Protection against streptococcal pharyngeal colonization with vaccines composed of M protein conserved regions," Advances in Experimental Medicine and Biology, vol. 303, pp. 159-167, 1991.

[27] T. L. Lamagni, J. Darenberg, B. Luca-Harari et al., "Epidemiology of severe Streptococcus pyogenes disease in Europe," Journal of Clinical Microbiology, vol. 46, no. 7, pp. 2359-2367, 2008.

[28] T. L. Lamagni, S. Neal, C. Keshishian et al., "Severe Streptococcus pyogenes infections, United Kingdom, 2003-2004," Emerging Infectious Diseases, vol. 14, no. 2, pp. 202-209, 2008.

[29] R. Kaul, A. McGeer, D. E. Low et al., "Population-based surveillance for group A streptococcal necrotiziug fasciitis: clinical features, prognostic indicators, and microbiologic analysis of seventy-seven cases," American Journal of Medicine, vol. 103, no. 1, pp. 18-24, 1997.

[30] P. Little, F. D. R. Hobbs, D. Mant, C. A. M. McNulty, and M. Mullee, "Incidence and clinical variables associated with streptococcal throat infections: a prospective diagnostic cohort study," British Journal of General Practice, vol. 62, no. 604, pp. e787-e794, 2012.

[31] A. Safar, D. Lennon, J. Stewart et al., "Invasive group a streptococcal infection and vaccine implications, Auckland, New Zealand," Emerging Infectious Diseases, vol. 17, no. 6, pp. 983989, 2011.

[32] C. Stockmann, K. Ampofo, A. L. Hersh et al., "Evolving epidemiologic characteristics of invasive group a streptococcal disease in utah, 2002-2010," Clinical Infectious Diseases, vol. 55, no. 4, pp. 479-487, 2012.

[33] J. R. Carapetis, A. C. Steer, and K. E. Mulholland, The Current Evidence for the Burden of Group A Streptococcal Diseases, World Health Organization, Geneva, Switzerland, 2005.

[34] P. N. Djomo, S. L. Thomas, and P. E. M. Fine, Correlates of Vaccine-Induced Protection: Methods and Implications, World Health Organization, Geneva, Switzerland, 2013.

[35] World Health Organization, Proceedings of the Tenth Global Vaccine Research Forum and Parallel Satellite Symposia, World Health Organization, Geneva, Switzerland, 2012.
[36] D. V. Madore, B. D. Meade, F. Rubin, C. Deal, and F. Lynn, "Utilization of serologic assays to support efficacy of vaccines in nonclinical and clinical trials: meeting at the crossroads," Vaccine, vol. 28, no. 29, pp. 4539-4547, 2010.

[37] D. Lennon, C. Jackson, S. Wong, M. Horsfall, J. Stewart, and S. Reid, "Fast tracking the vaccine licensure process to control an epidemic of serogroup B meningococcal disease in New Zealand," Clinical Infectious Diseases, vol. 49, no. 4, pp. 597-605, 2009.

[38] N. Andrews, R. Borrow, and E. Miller, "Validation of serological correlate of protection for meningococcal $\mathrm{C}$ conjugate vaccine by using efficacy estimates from postlicensure surveillance in England," Clinical and Diagnostic Laboratory Immunology, vol. 10, no. 5, pp. 780-786, 2003.

[39] R. Borrow, N. Andrews, D. Goldblatt, and E. Miller, "Serological basis for use of meningococcal serogroup $C$ conjugate vaccines in the United Kingdom: reevaluation of correlates of protection," Infection and Immunity, vol. 69, no. 3, pp. 1568-1573, 2001.

[40] E. Miller, D. Salisbury, and M. Ramsay, "Planning, registration, and implementation of an immunisation campaign against meningococcal serogroup C disease in the UK: a success story," Vaccine, vol. 20, supplement 1, pp. S58-S67, 2001.

[41] M. Granström and A. C. G. Voordouw, "Registration of influenza vaccines for children in Europe," Vaccine, vol. 29, no. 43, pp. 7572-7575, 2011.

[42] D. Goldblatt, M. Ramakrishnan, and K. O’Brien, "Using the impact of pneumococcal vaccines on nasopharyngeal carriage to aid licensing and vaccine implementation; A Pneumocarr meeting report March 27-28, 2012, Geneva," Vaccine, vol. 32, no. 1, pp. 146-152, 2013.

[43] D. F. Kelly, E. R. Moxon, and A. J. Pollard, "Haemophilus influenzae type b conjugate vaccines," Immunology, vol. 113, no. 2, pp. 163-174, 2004.

[44] J. B. Dale, T. A. Penfound, E. Y. Chiang, and W. J. Walton, "New 30 -valent $\mathrm{M}$ protein-based vaccine evokes cross-opsonic antibodies against non-vaccine serotypes of group A streptococci," Vaccine, vol. 29, no. 46, pp. 8175-8178, 2011.

[45] M. C. Hu, M. A. Walls, S. D. Stroop, M. A. Reddish, B. Beall, and J. B. Dale, "Immunogenicity of a 26-valent group A streptococcal vaccine," Infection and Immunity, vol. 70, no. 4, pp. 2171-2177, 2002.

[46] K. L. Kotloff, M. Corretti, K. Palmer et al., "Safety and immunogenicity of a recombinant multivalent group A streptococcal vaccine in healthy adults. Phase 1 trial," Journal of the American Medical Association, vol. 292, no. 6, pp. 709-715, 2004.

[47] M. R. Batzloff, H. Yan, M. Davies, J. Hartas, and M. Good, "Preclinical evaluation of a vaccine based on conserved region of M protein that prevents group A streptococcal infection," Indian Journal of Medical Research, vol. 119, supplement, pp. 104-107, 2004.

[48] T. A. Penfound, E. Y. Chiang, E. A. Ahmed, and J. B. Dale, "Protective efficacy of group A streptococcal vaccines containing type-specific and conserved M protein epitopes," Vaccine, vol. 28, no. 31, pp. 5017-5022, 2010.

[49] M. Zaman, A.-B. M. Abdel-Aal, Y. Fujita et al., "Immunological evaluation of lipopeptide group A streptococcus (GAS) vaccine: structure-activity relationship," PLoS ONE, vol. 7, no. 1, Article ID e30146, 2012.

[50] L. Guilherme, E. Postol, S. F. de Barros et al., "A vaccine against S. pyogenes: design and experimental immune response," Methods, vol. 49, no. 4, pp. 316-321, 2009. 
[51] C. M. Wernette, C. E. Frasch, D. Madore et al., "Enzyme-linked immunosorbent assay for quantitation of human antibodies to pneumococcal polysaccharides," Clinical and Diagnostic Laboratory Immunology, vol. 10, no. 4, pp. 514-519, 2003.

[52] N. F. Concepcion and C. E. Frasch, "Pneumococcal type 22F polysaccharide absorption improves the specificity of a pneumococcal-polysaccharide enzyme-linked immunosorbent assay," Clinical and Diagnostic Laboratory Immunology, vol. 8, no. 2, pp. 266-272, 2001.

[53] R. Bhushan, B. F. Anthony, and C. E. Frasch, "Estimation of group B streptococcus type III polysaccharide-specific antibody concentrations in human sera is antigen dependent," Infection and Immunity, vol. 66, no. 12, pp. 5848-5853, 1998.

[54] R. Bhushan and C. E. Frasch, "Estimation of group B streptococcus type Ia- and V-specific antibodies with different polysaccharide antigen preparations," in Proceedings of the 38th Interscience Conference on Antimicrobial Agents and Chemotherapy (ICAAC '98), Abstract G92, American Society for Microbiology, San Diego, Calif, USA, September 1998.

[55] H.-K. Guttormsen, C. J. Baker, M. H. Nahm et al., “Type III group B streptococcal polysaccharide induces antibodies that cross-react with Streptococcus pneumoniae type 14," Infection and Immunity, vol. 70, no. 4, pp. 1724-1738, 2002.

[56] D. R. Johnson, E. L. Kaplan, J. Sramek et al., Laboratory Diagnosis of Group A Streptococcal Infections, World Health Organization, Geneva, Switzerland, 1996.

[57] R. C. Lancefield, "Differentiation of group A streptococci with a common $\mathrm{R}$ antigen into three serological types, with special reference to the bactericidal test," Deutsche Medizinische Wochenschrift, vol. 83, no. 45, pp. 2018-2020, 1958.

[58] D. Goldblatt, B. D. Plikaytis, M. Akkoyunlu et al., "Establishment of a new human pneumococcal standard reference serum, 007sp," Clinical and Vaccine Immunology, vol. 18, no. 10, pp. 1728-1736, 2011.

[59] T. B. Martins, N. H. Augustine, and H. R. Hill, "Development of a multiplexed fluorescent immunoassay for the quantitation of antibody responses to group A streptococci," Journal of Immunological Methods, vol. 316, no. 1-2, pp. 97-106, 2006.

[60] E. H. Beachey, G. H. Stollerman, E. Y. Chiang, T. M. Chiang, J. M. Seyer, and A. H. Kang, "Purification and properties of $\mathrm{M}$ protein extracted from group A streptococci with pepsin: covalent structure of the amino terminal region of type $24 \mathrm{M}$ antigen," The Journal of Experimental Medicine, vol. 145, no. 6, pp. 1469-1483, 1977.

[61] S. Romero-Steiner, D. Libutti, L. B. Pais et al., "Standardization of an opsonophagocytic assay for the measurement of functional antibody activity against Streptococcus pneumoniae using differentiated HL-60 cells," Clinical and Diagnostic Laboratory Immunology, vol. 4, no. 4, pp. 415-422, 1997.

[62] H.-K. Guttormsen, Y. Liu, and L. C. Paoletti, "Functional activity of antisera to group B streptococcal conjugate vaccines measured with an opsonophagocytosis assay and HL-60 effector cells," Human Vaccines, vol. 4, no. 5, pp. 370-374, 2008.

[63] S. Romero-Steiner, C. Frasch, N. Concepcion et al., "Multilaboratory evaluation of a viability assay for measurement of opsonophagocytic antibodies specific to the capsular polysaccharides of Streptococcus pneumoniae," Clinical and Diagnostic Laboratory Immunology, vol. 10, no. 6, pp. 1019-1024, 2003.
[64] C. E. Rose, S. Romero-Steiner, R. L. Burton et al., "Multilaboratory comparison of Streptococcus pneumoniae opsonophagocytic killing assays and their level of agreement for the determination of functional antibody activity in human reference sera," Clinical and Vaccine Immunology, vol. 18, no. 1, pp. 135-142, 2011.

[65] M. H. Nahm and R. L. Burton, "Protocol for multiplexed opsonophagocytic killing assay (UAB-MOPA) for antibodies against Streptococcus pneumoniae," 2013, http://www.vaccine .uab.edu/UAB-MOPA.pdf.

[66] I. Feavers, I. Knezevic, M. Powell, and E. Griffiths, "Challenges in the evaluation and licensing of new pneumococcal vaccines, 7-8 July 2008, Ottawa, Canada," Vaccine, vol. 27, no. 28, pp. 36813688, 2009.

[67] S. A. Madhi, L. Kuwanda, C. Cutland, A. Holm, H. Käyhty, and K. P. Klugman, "Quantitative and qualitative antibody response to pneumococcal conjugate vaccine among African human immunodeficiency virus-infected and uninfected children," Pediatric Infectious Disease Journal, vol. 24, no. 5, pp. 410-416, 2005.

[68] M. Fabbrini, C. Sammicheli, I. Margarit et al., "A new flowcytometry-based opsonophagocytosis assay for the rapid measurement of functional antibody levels against Group B Streptococcus," Journal of Immunological Methods, vol. 378, no. 1-2, pp. 11-19, 2012.

[69] H.-K. Guttormsen, S. J. Mascuch, J. C. West, and L. C. Paoletti, "A fluorescence-based opsonophagocytosis assay to measure the functional activity of antibody to group B streptococcus," Human Vaccines, vol. 5, no. 7, pp. 461-466, 2009.

[70] A. C. Steer, I. Law, L. Matatolu, B. W. Beall, and J. R. Carapetis, "Global emm type distribution of group A streptococci: systematic review and implications for vaccine development," The Lancet Infectious Diseases, vol. 9, no. 10, pp. 611-616, 2009.

[71] P. R. Smeesters, D. J. McMillan, K. S. Sriprakash, and M. M. Georgousakis, "Differences among group A streptococcus epidemiological landscapes: consequences for M protein-based vaccines?" Expert Review of Vaccines, vol. 8, no. 12, pp. 17051720, 2009.

[72] A. P. Ralph and J. R. Carapetis, "Group A streptococcal diseases and their global burden," in Host-Pathogen Interactions in Streptococcal Diseases, vol. 368 of Current Topics in Microbiology and Immunology, pp. 1-27, 2012.

[73] M. Väkeväinen, W. Jansen, E. Saeland et al., "Are the opsonophagocytic activities of antibodies in infant sera measured by different pneumococcal phagocytosis assays comparable?" Clinical and Diagnostic Laboratory Immunology, vol. 8, no. 2, pp. 363-369, 2001.

[74] J. E. Martinez, E. A. Clutterbuck, H. Li, S. Romero-Steiner, and G. M. Carlone, "Evaluation of multiplex flow cytometric opsonophagocytic assays for determination of functional anticapsular antibodies to Streptococcus pneumoniae," Clinical and Vaccine Immunology, vol. 13, no. 4, pp. 459-466, 2006.

[75] J. E. Martinez, S. Romero-Steiner, T. Pilishvili et al., "A flow cytometric opsonophagocytic assay for measurement of functional antibodies elicited after vaccination with the 23 -valent pneumococcal polysaccharide vaccine," Clinical and Diagnostic Laboratory Immunology, vol. 6, no. 4, pp. 581-586, 1999.

[76] K. T. Bieging, G. Rajam, P. Holder, R. Udoff, G. M. Carlone, and S. Romero-Steiner, "Fluorescent multivalent opsonophagocytic assay for measurement of functional antibodies to Streptococcus pneumoniae," Clinical and Diagnostic Laboratory Immunology, vol. 12, no. 10, pp. 1238-1242, 2005. 
[77] M. H. Nahm, D. E. Briles, and X. Yu, "Development of a multispecificity opsonophagocytic killing assay," Vaccine, vol. 18, no. 24, pp. 2768-2771, 2000.

[78] R. L. Burton and M. H. Nahm, "Development and validation of a fourfold multiplexed opsonization assay (MOPA4) for pneumococcal antibodies," Clinical and Vaccine Immunology, vol. 13, no. 9, pp. 1004-1009, 2006.

[79] D. Bogaert, M. Sluijter, R. De Groot, and P. W. M. Hermans, "Multiplex opsonophagocytosis assay (MOPA): a useful tool for the monitoring of the 7-valent pneumococcal conjugate vaccine," Vaccine, vol. 22, no. 29-30, pp. 4014-4020, 2004.

[80] S. Romero-Steiner, C. E. Frasch, G. Carlone, R. A. Fleck, D. Goldblatt, and M. H. Nahm, "Use of opsonophagocytosis for serological evaluation of pneumococcal vaccines," Clinical and Vaccine Immunology, vol. 13, no. 2, pp. 165-169, 2006.

[81] K. H. Kim, J. Yu, and M. H. Nahm, "Efficiency of a pneumococcal opsonophagocytic killing assay improved by multiplexing and by coloring colonies," Clinical and Diagnostic Laboratory Immunology, vol. 10, no. 4, pp. 616-621, 2003.

[82] Y. Ji, B. Carlson, A. Kondagunta, and P. P. Cleary, "Intranasal immunization with $\mathrm{C} 5 \mathrm{a}$ peptidase prevents nasopharyngeal colonization of mice by the group A streptococcus," Infection and Immunity, vol. 65, no. 6, pp. 2080-2087, 1997.

[83] H. S. Park and P. P. Cleary, "Active and passive intranasal immunizations with streptococcal surface protein C5a peptidase prevent infection of murine nasal mucosa-associated lymphoid tissue, a functional homologue of human tonsils," Infection and Immunity, vol. 73, no. 12, pp. 7878-7886, 2005.

[84] A. L. Bisno, F. A. Rubin, P. P. Cleary, and J. B. Dale, "Prospects for a group A streptococcal vaccine: rationale, feasibility, and obstacles-report of a National Institute of Allergy and Infectious Diseases workshop," Clinical Infectious Diseases, vol. 41, no. 8, pp. 1150-1156, 2005.

[85] A. Henningham, C. M. Gillen, and M. J. Walker, "Group a streptococcal vaccine candidates: potential for the development of a human vaccine," Current Topics in Microbiology and Immunology, vol. 368, pp. 207-242, 2013.

[86] W. A. Hayman, E. R. Brandt, W. A. Relf, J. Cooper, A. Saul, and M. F. Good, "Mapping the minimal murine T cell and B cell epitopes within a peptide vaccine candidate from the conserved region of the M protein of group A streptococcus," International Immunology, vol. 9, no. 11, pp. 1723-1733, 1997.

[87] J. M. Skinner, I. C. Caro-Aguilar, A. M. Payne, L. Indrawati, J. Fontenot, and J. H. Heinrichs, "Comparison of rhesus and cynomolgus macaques in a Streptococcus pyogenes infection model for vaccine evaluation," Microbial Pathogenesis, vol. 50, no. 1, pp. 39-47, 2011.

[88] M. Saaka, B. J. Okoko, R. C. Kohberger et al., "Immunogenicity and serotype-specific efficacy of a 9-valent pneumococcal conjugate vaccine (PCV-9) determined during an efficacy trial in the Gambia," Vaccine, vol. 26, no. 29-30, pp. 3719-3726, 2008.

[89] J. Taranger, B. Trollfors, T. Lagergård et al., "Correlation between pertussis toxin IgG antibodies in postvaccination sera and subsequent protection against pertussis," Journal of Infectious Diseases, vol. 181, no. 3, pp. 1010-1013, 2000.

[90] C. J. Baker, V. J. Carey, M. A. Rench et al., "Maternal antibody at delivery protects neonates from early onset group B streptococcal disease," Journal of Infectious Diseases, vol. 209, no. 5, pp. 781-788, 2014.

[91] S. P. O’Connor, D. Darip, K. Fraley, C. M. Nelson, E. L. Kaplan, and P. P. Cleary, "The human antibody response to streptococcal
C5a peptidase," The Journal of Infectious Diseases, vol. 163, no. 1, pp. 109-116, 1991.

[92] H. Sabharwal, F. Michon, D. Nelson et al., "Group A streptococcus (GAS) carbohydrate as an immunogen for protection against GAS infection," Journal of Infectious Diseases, vol. 193, no. 1, pp. 129-135, 2006.

[93] A. L. Gould, "Timing of futility analyses for 'proof of concept' trials," Statistics in Medicine, vol. 24, no. 12, pp. 1815-1835, 2005.

[94] E. N. Fox, R. H. Waldman, M. K. Wittner, A. A. Mauceri, and A. Dorfman, "Protective study with a group A streptococcal M protein vaccine. Infectivity challenge of human volunteers," Journal of Clinical Investigation, vol. 52, no. 8, pp. 1885-1892, 1973.

[95] S. M. Polly, R. H. Waldman, P. High, M. K. Wittner, and A. Dorfman, "Protective studies with a group A streptococcal M protein vaccine. II. Challenge of volunteers after local immunization in the upper respiratory tract," Journal of Infectious Diseases, vol. 131, no. 3, pp. 217-224, 1975.

[96] R. D’Alessandri, G. Plotkin, R. M. Kluge et al., "Protective studies with group A streptococcal M protein vaccine. III. Challenge of volunteers after systemic or intranasal immunization with type 3 or type 12 group A Streptococcus," The Journal of Infectious Diseases, vol. 138, no. 6, pp. 712-718, 1978.

[97] R. Edelman, S. S. Wasserman, S. A. Bodison, J. G. Perry, M. O’Donnoghue, and L. J. DeTolla Jr., "Phase II safety and immunogenicity study of type $\mathrm{F}$ botulinum toxoid in adult volunteers," Vaccine, vol. 21, no. 27-30, pp. 4335-4347, 2003.

[98] D. M. Ferreira, D. R. Neill, M. Bangert et al., "Controlled human infection and rechallenge with Streptococcus pneumoniae reveals the protective efficacy of carriage in healthy adults," The American Journal of Respiratory and Critical Care Medicine, vol. 187, no. 8, pp. 855-864, 2013.

[99] C. O. Tacket, M. B. Cohen, S. S. Wasserman et al., "Randomized, double-blind, placebo-controlled, multicentered trial of the efficacy of a single dose of live oral cholera vaccine CVD 103$\mathrm{HgR}$ in preventing cholera following challenge with Vibrio cholerae O1 El tor inaba three months after vaccination," Infection \& Immunity, vol. 67, no. 12, pp. 6341-6345, 1999.

[100] R. W. Sauerwein, M. Roestenberg, and V. S. Moorthy, "Experimental human challenge infections can accelerate clinical malaria vaccine development," Nature Reviews Immunology, vol. 11, no. 1, pp. 57-64, 2011.

[101] W. Sun, K. H. Eckels, J. R. Putnak et al., "Experimental dengue virus challenge of human subjects previously vaccinated with live attenuated tetravalent dengue vaccines," Journal of Infectious Diseases, vol. 207, no. 5, pp. 700-708, 2013. 


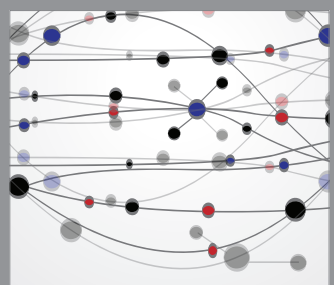

The Scientific World Journal
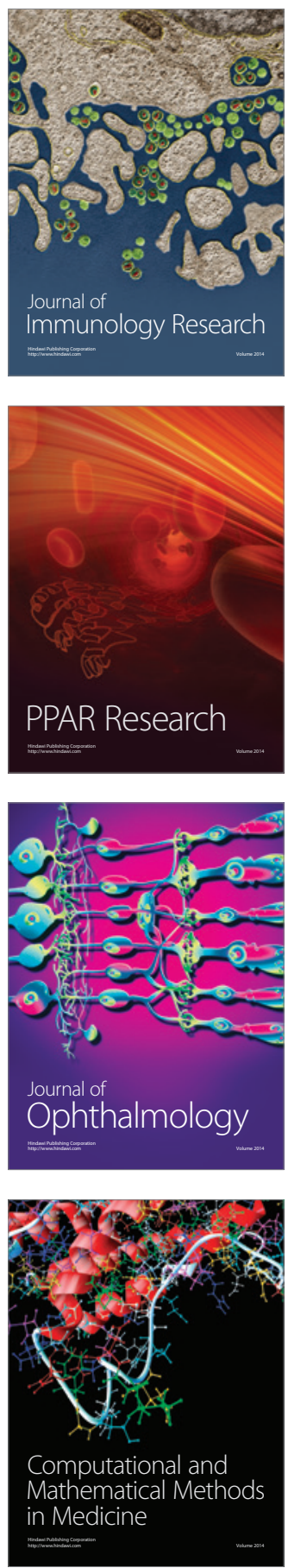

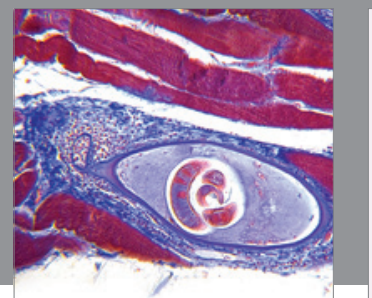

Gastroenterology

Research and Practice
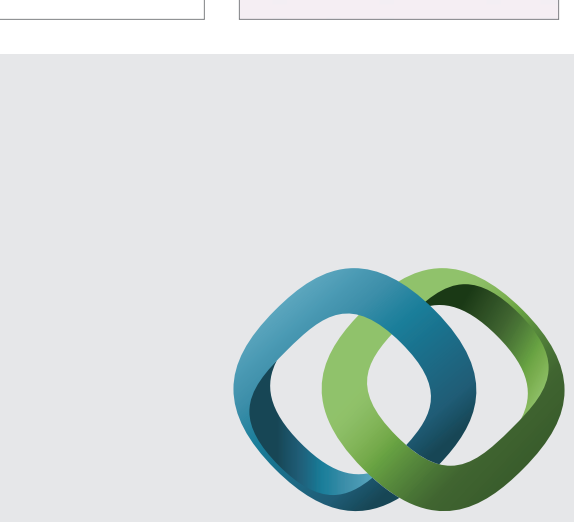

\section{Hindawi}

Submit your manuscripts at

http://www.hindawi.com
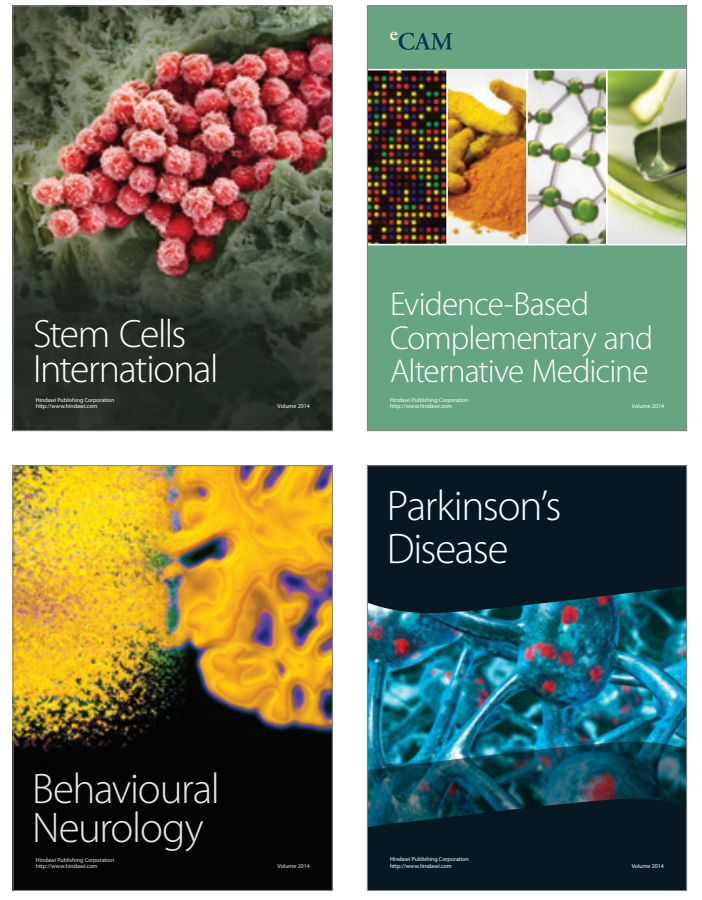
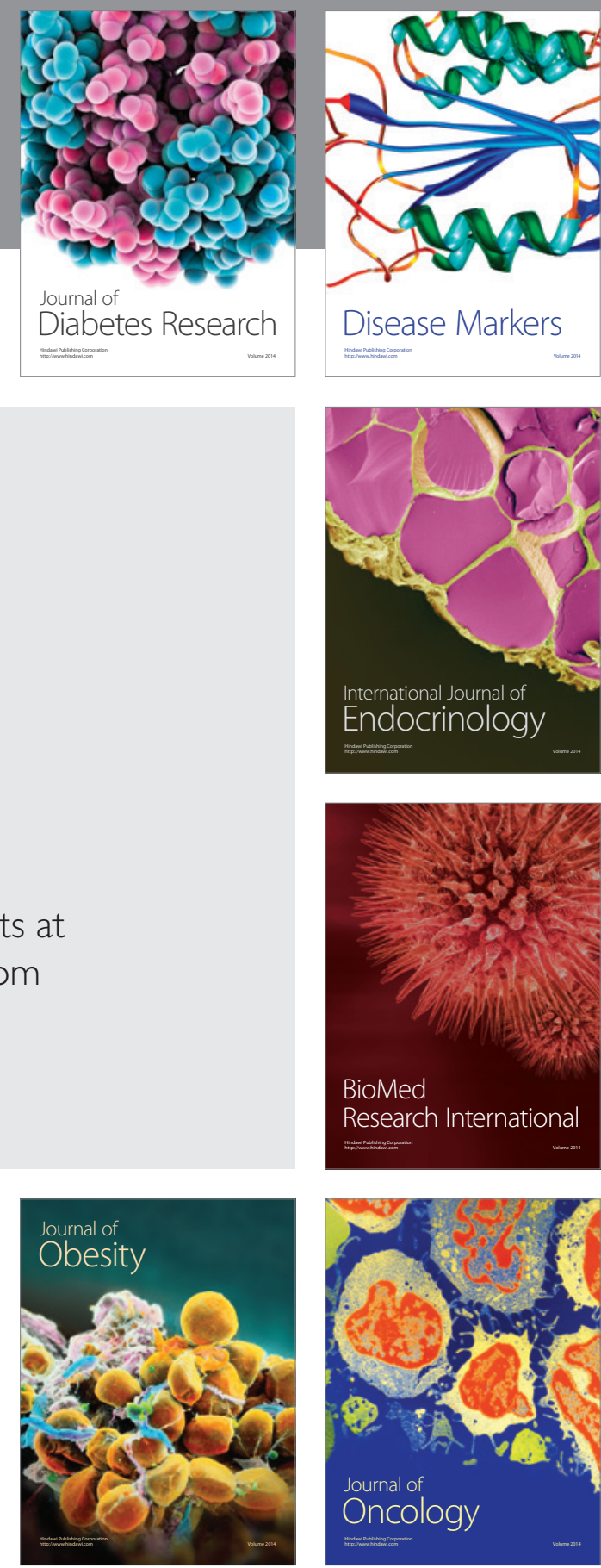

Disease Markers
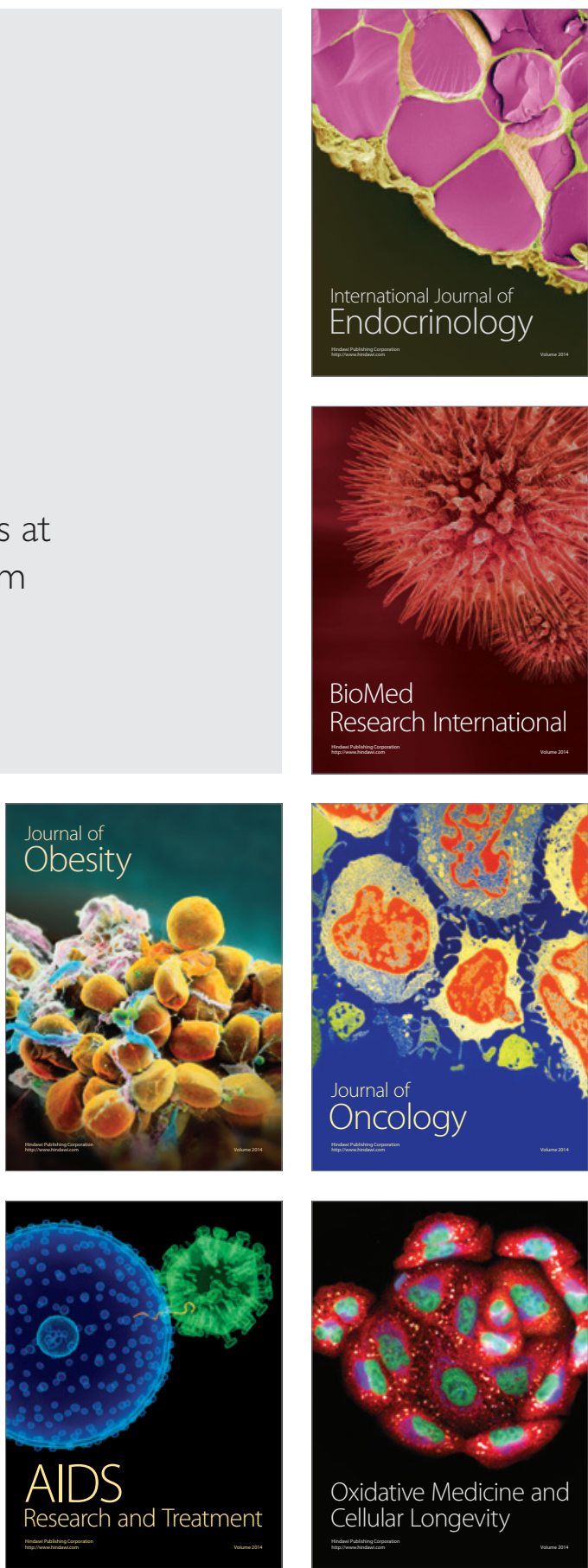